\title{
Sistemas de Gestión de Contenidos (SGC): una revisión sistemática de literatura
}

\author{
Víctor Daniel Gil Vera \\ Fundación Universitaria Luis Amigó - Centro de Investigaciones Religiosos Terciarios Capuchinos \\ victor.gilve@amigo.edu.co
}

[Tipo de Artículo: Revisión. Recibido el 20/04/2015. Aprobado el 22/07/2015]

\begin{abstract}
Resumen. Los sistemas de gestión de contenidos (SGC) facilitan la creación y desarrollo de aplicaciones web. En la actualidad, gracias a la diversidad de programas y herramientas de código fuente abierto, tareas que requerían conocimientos y técnicas avanzadas de programación pueden hacerse con facilidad y en poco tiempo, facilitando el trabajo a profesionales y desarrolladores de software. Este trabajo es un resultado del proyecto de investigación "Sistema de información y Base de datos unificada para la población atendida Provincia San José en los diversos colectivos de los Religiosos Terciarios Capuchinos" cuyo objetivo principal era diseñar e implementar un sistema de información orientado a la web mediante la implementación de un sistema de gestión de contenidos (SGC), que permitiera gestionar la información de la población atendida en los centros de atención especializados (CAE) para menores infractores, de una manera integrada y unificada. Con el desarrollo de este trabajo se concluye que los sistemas de gestión de contenidos (SGC) facilitan la creación de sitios y aplicaciones web, la gestión y administración de la información gracias a su versatilidad y fácil implementación.
\end{abstract}

Palabras clave. Revisión Sistemática de Literatura, SGC, Aplicaciones Web, Desarrollo de Software.

\section{Content Management Systems (CMS): a systematic literature review}

Abstract. Content Management Systems (CMS) for the development of web applications make easier the work of developers and professionals in computing industry. Nowadays, because of the plethora of online tools and technologies for managing web applications the tasks that otherwise required knowledge and advanced programming techniques now can be performed in a short time. The development of this work results from the research project called "Information System and Unified Database for the population Provincia de San José in the different groups of the Religious Tertiary Capuchins", which results from the need to create a web application that make easier the management of the information coming from the various centers of rehabilitation of young offenders. The Content Management Systems (CMS) make easier the management of information because of their versatility and easy deployment.

Keywords. Systematic Literature Review; CMS; Web applications; Software Development.

\section{INTRODUCCIÓN}

La gran popularidad, versatilidad, uso y aplicación de los sistemas de gestión de contenidos (SGC), han transformado la manera tradicional de desarrollar sitios y aplicaciones web, la cual se basa en lenguajes avanzados de programación. La "Provincia de San José" administrada por la Orden de Religiosos Terciarios Capuchinos (CTC), lleva a cabo la gestión y administración de los diferentes centros de atención especializados para la rehabilitación de menores infractores en Colombia. Considerando los grandes volúmenes de información que manejan los diferentes centros y la alta complejidad del proceso de generación de indicadores y reportes estadísticos, se desarrolló el aplicativo web "Amigonianos Provincia San José" con un WCMS (Sistemas de Gestión de Contenidos Web), el cual es utilizado en las diferentes sedes pertenecientes a la Provincia San José.

Este trabajo se divide como sigue: en la primera parte se presenta la metodología utilizada para la realización de la revisión sistemática de literatura (RSL), en la segunda los resultados obtenidos de la revisión, en la tercera se comparan los diferentes sistemas de gestión de contenidos (SGC) más utilizados a nivel mundial encontrados en la revisión y en la última las conclusiones y recomendaciones finales.
Con la realización de este trabajo se concluye que los sistemas de gestión de contenidos (SGC) facilitan la gestión y administración de la información gracias a su versatilidad y fácil implementación.

\section{REVISIÓN SISTEMÁTICA DE LITERATURA}

Una RSL es un medio para identificar, evaluar e interpretar toda la investigación pertinente disponible relacionada con una pregunta de investigación, área temática o fenómeno de interés. Los estudios individuales que sirven de base para la realización de una RSL se denominan estudios primarios, la RSL por si sola es considerada como un estudio secundario [1]. La metodología general para la realización de una RSL definida por [1] es:

- Identificación de investigación: El objetivo de una revisión sistemática es encontrar la mayor cantidad posible de estudios primarios relacionados con una pregunta de investigación, tomando como base criterios y parámetros de búsqueda antes de hacer la selección final. La definición de estos criterios es la principal diferencia que la distingue de la revisión tradicional de literatura.

- Selección de estudios: Una vez obtenidos los estudios primarios potencialmente relevantes, se debe evaluar su calidad real, para filtrar los estudios que realmente 
puedan contribuir a responder la(s) pregunta(s) de investigación.

- Extracción de datos: El objetivo de esta etapa es diseñar formularios de extracción de datos para registrar con precisión la información que los investigadores obtienen de los estudios primarios. Los formularios de extracción de datos deben ser definidos al momento de definir el protocolo de estudio.
- Síntesis: Consiste en recopilar y resumir los resultados de los estudios primarios seleccionados. La síntesis debe ser descriptiva (no cuantitativa). Sin embargo, a veces es posible complementar una síntesis descriptiva con un resumen cuantitativo

A continuación, se presentan las tres etapas principales relacionadas con la búsqueda, depuración y selección de información:

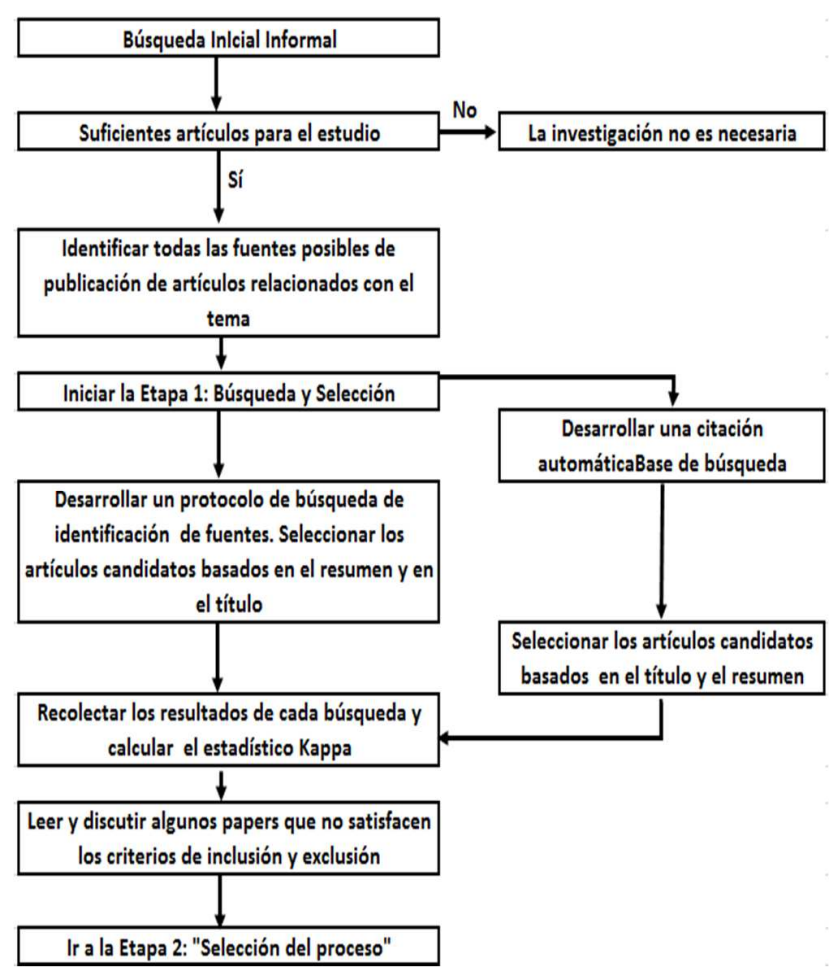

Fig 6. Etapa 1 (RSL). Fuente: [1]

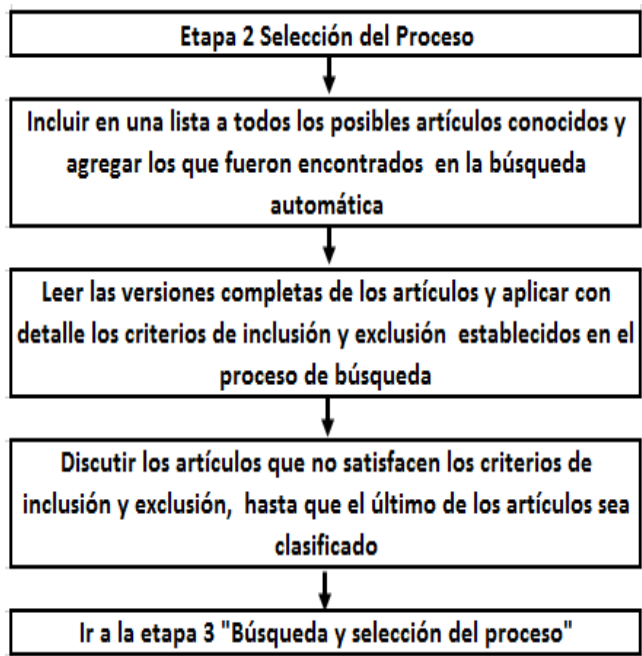

Fig 2. Etapa 2 (RSL). Fuente: [1] 


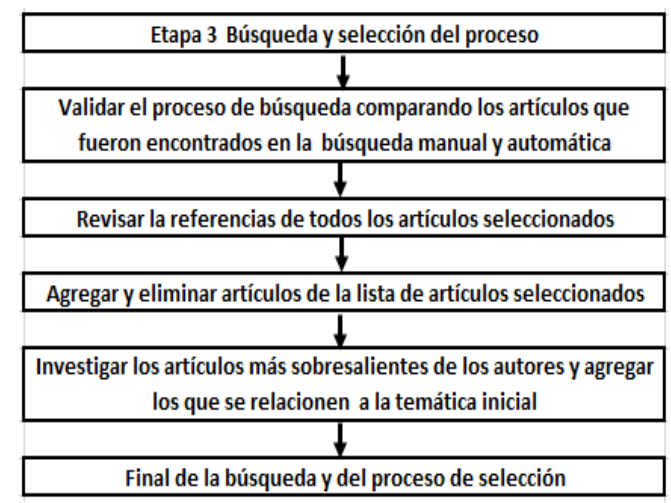

Fig 3. Etapa 3 (RSL). Fuente: [1]

\section{SISTEMAS DE GESTIÓN DE CONTENIDOS}

A continuación, se presentan algunas definiciones generales de los sistemas de gestión de contenidos (SGC):

- Los SGC son sistemas que combinan una gama de gestión temática y herramientas pedagógicas para proporcionar un medio para el diseño y construcción de entornos virtuales de aprendizaje [1]

- Los SGC son aplicaciones de software para la creación, publicación, edición y gestión de contenido. Son ampliamente utilizados por la agencias de noticias y medios de comunicación, sitios web de comercio electrónico, bibliotecas, radiodifusión, industria cinematográfica e instituciones educativas para manejar el contenido de manera eficiente [2]

- Los SGC son sistemas basados en la web, que organizan el contenido de la información [4].

En la Fig 4, se presentan las etapas necesarias para la creación de un SGC:

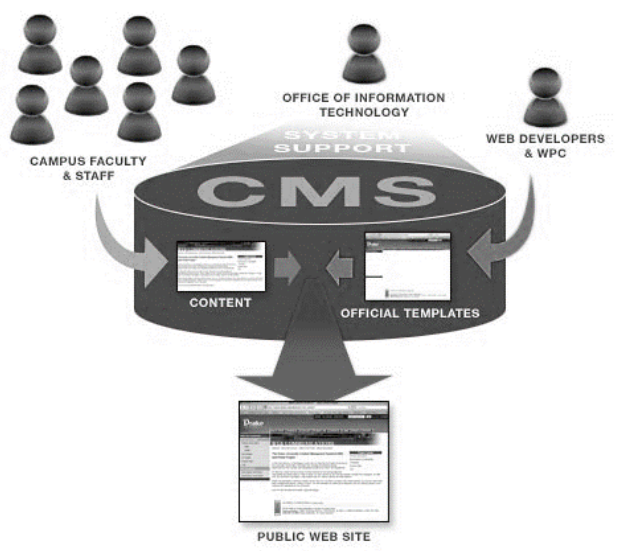

Figura 4. Ciclo SGC. Fuente: [1]

En primer lugar, la facultad o cuerpo administrativo organizacional suministra el contenido a la oficina de información tecnológica, posteriormente, el desarrollador web (personal encargado) selecciona la plantilla oficial para la creación del sitio web final. Los SGC se clasifican en:

- Sistemas de Gestión de Contenidos Web (W-SGC)

- Sistemas de Gestión de Contenidos para el Aprendizaje (L-SGC)

- Sistemas de Gestión de Contenidos Transaccionales (T-SGC)

- Sistemas de Gestión de Contenidos Integrados (ISGC)

- Sistemas de Gestión de Contenidos para Publicaciones (P-SGC)

- Sistemas de Gestión de Contenidos para Empresas (E-SGC)

\subsection{Sistemas de Gestión de Contenidos Web (W- SGC)}

Un sistema de gestión de contenidos web (W-SGC) es un sistema que permite a usuarios con pocos o nulos conocimientos de programación, desarrollar rápidamente sitios web, crear, editar y publicar contenidos en el mismo [4]. Gracias a las grandes ventajas que disponen este tipo de sistemas de gestión de contenidos y a la alta disponibilidad de funciones potentes y fiables de código abierto, el número de sitios web construidos con W-SGS se ha incrementado a nivel mundial [4].

Según [4] las principales ventajas de los W-SGC son:

- Permiten generar automáticamente la estructura de navegación web y organización del contenido

- Cuentan con herramientas para la edición enriquecida de texto WYSIWYG

- Disponen de una amplia variedad de plantillas que permiten cambiar fácilmente temas de presentación personalizados

- Facilitan la gestión de la seguridad, inicio de sesión y control de acceso basado en roles

- Modularidad, permiten añadir módulos adicionales y plug-ins.

En un W-SGC toda la administración del contenido se realiza a través de formularios web con un explorador Web. Los usuarios únicamente se limitan a copiar y pegar el contenido existente o a llenar los espacios en blanco en 
un formulario [4]. Un W-SGC admite la creación y publicación de contenido estructurado en formatos Web, como HTML, XHTML, XML y PDF [6].

\subsection{Sistemas de Gestión de Contenidos de Aprendizaje (L-SGC)}

Los L-SGC, se definen como:

- Los L-SGC son la versión corporativa de los sistemas tradicionales de gestión de cursos académicos desarrollados inicialmente para la educación superior. Están diseñados para permitir a maestros y docentes con poca experiencia en tecnología, diseñar, crear, entregar y medir los resultados de cursos virtuales rápidamente [5].

- Los L-SGC son los sistemas más populares de eLearning existentes en la actualidad, que incorporan la gestión de contenidos para el sistema de gestión académica del estudiante (registro, comunicación, notas, tutoriales) [6]. Los L-SGC permiten organizar el contenido en relación con los temas de un curso. La estructura de estos temas puede presentar un formato de lista o un formato de índice. Por lo general, el contenido de las asignaturas consta de recursos de aprendizaje y las actividades elegidas y secuenciados por la facultad de acuerdo al diseño del curso, a la que cada estudiante debe adaptarse [7].

El término L-SGC fue creado por un consorcio de fabricantes de software para diferenciarlos de los sistemas de gestión de aprendizaje tradicionales (SGL). A pesar de que los sistemas de gestión de contenidos de aprendizaje (L-SGC) y los sistemas de gestión de aprendizaje ( $S G L)$ comparten nombres similares tienen diferentes funcionalidades [8]. Los L-SGC se centran en el contenido mientras que los SGL se centran en los estudiantes. La función principal de un SGL es gestionar el proceso de aprendizaje de un estudiante. Este proceso suele incluir la identificación de una necesidad, como el seguimiento de actividades, presentación de pruebas, informes, análisis, entre otros [7].

\subsection{Sistemas de Gestión de Contenidos de Transaccionales (T-CMS)}

Son definidos como sistemas que permiten a las organizaciones capturar, procesar, y acceder a imágenes electrónicas de los documentos existentes en una plataforma de gestión de contenidos unificada [8]. El contenido de estos sistemas puede incluir recursos y documentos electrónicos, fotos, informes, reportes generados por ordenador, datos $\mathrm{XML}$, formularios electrónicos, entre otros, los cuales se gestionan adecuadamente y se integran con los sistemas basados en datos de línea de negocio [8].

\subsection{Sistemas de Gestión de Contenidos Integrados (I-CMS)}

Son sistemas que permiten gestionar todo el contenido web sin necesidad de poseer conocimientos avanzados de HTML, permitiendo a profesionales de diferentes áreas (marketing, gestión de productos, ingeniería, ventas, atención al cliente, etc.) crear y modificar fácilmente su contenido, cambiar y mejorar mensajes publicitarios, introducir nuevos productos, etc., lo que les da la flexibilidad para controlar y adoptar estrategias según las necesidades del mercado, permitiéndoles asumir retos empresariales [9].

\subsection{Sistemas de Gestión de Contenidos Empresariales (E-CMS)}

Los E-SGC son sistemas que permiten realizar una gestión integrada de la información no estructurada de una empresa: documentos, correos electrónicos, portales web y activos digitales [10]. En otras palabras, un sistema de gestión de contenidos empresariales permite gestionar un gran número de documentos seguros para muchas organizaciones [11]. Además de evitar la saturación de información y las altas inversiones relacionadas con costos de publicaciones web, los E-SGC facilitan la toma de decisiones y permiten tener una mayor eficiencia de intercambio de información y colaboración compartida, control de cumplimiento y reutilización de contenidos para múltiples medios de comunicación [12].

\section{METODOLOGÍA}

Para la construcción de este trabajo se empleó la metodología de la revisión sistemática de literatura (RSL), que es definida como un proceso de investigación riguroso, auditable y sistemático, cuyo objetivo principal es responder a una o más preguntas de investigación, tomando como base literatura científica, literatura gris y publicaciones electrónicas especializadas [13].

Se realizó una revisión de artículos, libros, artículos de conferencias, artículos en desarrollo, resúmenes y publicaciones web especializadas. Las bases de datos utilizadas fueron: Scopus, ACM IEEE Xplore, Isis. Las palabras clave utilizadas fueron: content management systems, enterprise, transactional, learning, integrated, publications, web development, online applications. En total, se recolectaron 79 publicaciones. El rango de fechas estuvo comprendido desde el año 2008 hasta la presente. La pregunta de investigación sobre la cual se basó la revisión fue:

P1.¿Cuáles son las principales características de los sistemas de gestión de contenidos SGC para la creación y desarrollo de sitios y aplicaciones web?. Con esta pregunta se pretende identificar las principales características de los SGC (L-SGC, T-SGC, W-SGC, PSGC, T-SGC, I-SGC) más utilizados en la actualidad para el desarrollo de sitios y aplicaciones web.

La ecuación de búsqueda utilizada fue:

(TITLE-ABS-KEY (CMS)) OR $\quad((((($ content $)) \quad$ AND (management)) AND (systems)) AND (web)) AND ((applications) OR (enterprise) OR (transactional) OR (learning) OR (integrated) OR (publications)) > 2008 


\section{RESULTADOS}

El año y el autor con el mayor número publicaciones en el tema según los resultados de la búsqueda fueron: 2010 y Clarebout, G., respectivamente. En la Tabla 1 y Tabla 2, se presentan las publicaciones por año y por tipo de documento:

TABLA 1

Publicaciones por Año

\begin{tabular}{ll}
\hline Año & Cantidad \\
\hline 2014 & 8 \\
2013 & 11 \\
2012 & 14 \\
2011 & 9 \\
2010 & 15 \\
2009 & 11 \\
2008 & 11 \\
Total & 79 \\
\hline \multicolumn{2}{c}{ Número de publicaciones por tipo } \\
\hline Tipo de Documento & Cantidad \\
Artículos de conferencias & 40 \\
Artículos & 26 \\
Resúmenes de conferencias & 6 \\
Capítulos de libros & 4 \\
Resúmenes & 2 \\
Articulos en desarrollo & 1 \\
Total & 79 \\
\hline
\end{tabular}

\section{DISCUSIÓN}

En esta sección se presentan los principales SGC encontrados en la RSL:

- Joomla: Es uno de los más completos y sofisticados sistemas de gestión de contenidos. Es utilizado principalmente para crear sitios web multilingues altamente interactivos como portales online para comunidades, blogs, aplicaciones para comercio electrónico, entre otros [14].

- Drupal: Plataforma dinámica de código abierto para la construcción de sitios web, flexibles y robustos. Permite al usuario actualizar páginas web sin tener que poseer conocimientos técnicos avanzados ni tener que asegurar que se ajusta el flujo de trabajo de su organización. Puede ser instalada en varios idiomas, lo que permite tanto a los administradores y usuarios ver un sitio web en su propio idioma [15].

- Wordpress: Fue diseñado inicialmente como una plataforma para blogs, sin embargo, en los últimos años se ha transformado a un moderno sistema de gestión de contenidos. Una de sus principales ventajas es la gran cantidad de plug-ins que dispone, que permiten mejorar la funcionalidad de la interfaz del usuario [15]. De hecho, todos los aspectos de los sitios web que hacen referencia a la creación, organización y optimización de motores de búsqueda, pueden ser gestionados por el usuario [16].

- Webnode: Sistema de creación de páginas web, revolucionario interactivo y gratuito que permite crear sitios web en tiempo real, siguiendo el mecanismo práctico de arrastrar y soltar. Los usuarios pueden crear, diseñar, desarrollar sitios y aplicaciones web de principio a fin. Está diseñado para ser utilizado de forma rápida y fácil. Permite crear y diseñar sitios web de alta calidad en poco tiempo [17].

- WebGui: Plataforma para la creación de sitios y aplicaciones web, que permiten una fácil gestión de contenidos, manteniendo al mismo tiempo la capacidad de crear e instalar aplicaciones personalizadas. Permite publicar artículos, participar en foros, crear galerías de fotos, realizar encuestas y sondeos, gestionar proyectos, crear calendarios de eventos interactivos, crear formularios de entrada de datos complejos, vender y anunciar productos, vender y mantener servicios de suscripción, mantener la seguridad del sitio a través de usuarios y grupos, gestionar los niveles de uso y seguridad [18].

- Rubedo: Es un SGC para la gestión de contenidos de código abierto, basado en la plataforma (NoSQL), que permite crear múltiples sitios web [19]. Se caracteriza por su flexibilidad y por proporcionar a los usuarios bases sólidas para construir una estrategia web integral (páginas web, sitios web para móviles, aplicaciones de negocios, extranet, comercio, entre otras). Su rendimiento y agilidad han sido aprobados por muchos clientes en todo el mundo [19].

- Modx: Es un (SGC) y Framework integrado. A pesar de las posibilidades ilimitadas y variedad de recursos que MODX brinda a los usuarios, es un sistema intuitivo para trabajar. Se caracteriza principalmente por ofrecer libertad creativa a los usuarios [20].

- X3CMS: Sistema de gestión de contenidos sofisticado y completo que cuenta con un núcleo de MVC (ModeloVista-Controlador) que proporciona características inesperadas para crear fácilmente sitios y aplicaciones web fascinantes y complejas. X3CMS permite crear sitios web impresionantes. Es uno de los SGC mejor calificados y usados en el mundo [21]. A pesar de que no cuenta con herramientas de gestión de documentos, cuenta con buenas herramientas para la gestión y seguimiento de proyectos [21].

- EZ Publish Platform: Sistema de gestión de contenidos diseñado para construir, gestionar y ejecutar sitios web exigentes que permitan afrontar los retos del mercado. Tiene la capacidad de suministrar el contenido de manera personalizada a diferentes clases de públicos y ofrece herramientas de medición para seguir y adaptarse a los comportamientos de los usuarios [22], que la convierten en una plataforma 
completa para que las organizaciones puedan construir sus propias experiencias digitales [22].

- Expression Engine: Sistema de gestión de contenidos multipropósito, desarrollada por la empresa de software estadounidense EllisLab. Se trata de una plataforma web modular que ofrece tres niveles de licencias: comercial, no comercial y freelancer [23]. Una de sus principales características es su facilidad de uso. En comparación con otros SGC, ha demostrado ser una solución versátil, que incluye un robusto motor de SGC, y herramientas sofisticadas para el comercio electrónico [23].

- Movable Type: Plataforma para la creación de sitios web considerado como el SGC más utilizado en el mundo. Permite la construcción de blogs, páginas web con un alto grado de interactividad, redes sociales, entre otras. [24]. Dispone de un número de blogs ilimitado, cuenta con un sofisticado sistema de comentarios que permiten a los usuarios interacturar directamente entre ellos y con el administrador del sitio [24].

- Radiant CMS: Sistema de gestión de contenidos de código abierto, diseñado para pequeños equipos de trabajo. Es similar a Textpattern o MovableType, pero es considerado como un sistema de gestión de contenidos de propósito general [25]. Fue construido con Ruby on Rails y es de libre distribución y uso [25].

- Silver Stripe: Sistema de gestión de contenidos de código fuente abierto (SGC) para la creación y mantenimiento de sitios web. Cuenta con un panel de administración web que permite a los usuarios realizar modificaciones en partes específicas de sus sitios, que incluye un editor web WYSIWYG, fue construido con PHP [25].

La Tabla 3 presenta los principales SGC utilizados en la industria de la computación:

TABLA 3

Principales CMS y Año de Fundación

\begin{tabular}{ll}
\multicolumn{2}{c}{ Principales CMS y Año de Fundación } \\
\hline CMS & Año \\
\hline Joomla & 2005 \\
Drupal & 2001 \\
WordPress & 2003 \\
WebNode & 2004 \\
WebGUI & 2008 \\
Rubedo & 2008 \\
MODX & 2014 \\
Concrete5 & 2006 \\
X3 CMS & 2004 \\
Movable Type & 2001 \\
Silver Stripe & 2000 \\
\hline
\end{tabular}

\section{CONCLUSIONES}

El desarrollo de sitios y aplicaciones web con sistemas de gestión de contenidos SGC facilitan el trabajo de desarrolladores y profesionales de la industria de la computación. En la actualidad, gracias a la diversidad de herramientas tecnológicas de código fuente abierto para la gestión y administración de aplicaciones web, tareas que requerían conocimientos avanzados de programación pueden realizarse en poco tiempo y sin mayor esfuerzo.

La principal ventaja de los SGC es que son de libre acceso y no representan costos relacionados con la compra de licencias, administración o soporte. La mayoría de ellos cuenta con un conjunto amplio de herramientas que facilitan la administración y gestión de la información

A diferencia del software propietario para la creación de sitios y aplicaciones web, los SGC permiten conocer y manipular el código fuente, lo que permite al usuario realizar modificaciones y corregir errores sin tener que depender de proveedores ni del lanzamiento de nuevas versiones.

\section{AGRADECIMIENTOS}

A todos los docentes de la "Fundación Universitaria Luis Amigó" y al "Centro de Investigaciones Provincia San José", cuyos aportes y contribuciones hicieron posible el desarrollo de este trabajo.

\section{REFERENCIAS}

[1] Kitchenham, B. "Procedures for performing systematic reviews". Keele, UK, Keele University, 33(1), pp.1-26, 2004.

[2] H. Coates, R. James, and G. Baldwin, "A critical examination of the effects of learning management systems on university teaching and learning," Springer, Ed. 2005, pp. 19-36.

[3] G. B. Laleci, G. Aluc, a. Dogac, a. Sinaci, O. Kilic, and F. Tuncer, "A semantic backend for content management systems," KnowledgeBased Syst., vol. 23, no. 8, pp. 832-843, Dec. 2010.

[4] D. Distante, M. Risi, and G. Scanniello, "Extending Web Content Management Systems Navigation Capabilities with Semantic Navigation Maps," Int. Symp. Web Syst. Evol., pp. 1-5, 2010.

[5] W. Powel and C. Gill, "Web Content Management Systems in Higher Education," no. 2, pp. 43-50, 2003.

[6] M. Meike, "Security in Open Source Web Content Management Systems," IEEE Internet Secur. Priv., no. August, pp. 40-48, 2009.

[7] B. S. R. Robbins, "The Evolution of the Learning Content Management System," pp. 1-4, 2007.

[8] M. Sein, F. García, and M. Condez, "Learning content management systems for the definition of adaptive learning environments," pp. 105-110, 2014.

[9] Y. Akp and Ş. Hüseyin, "Development of a Learning Content Management System Based on Interactive Learning Object Approach," pp. 5-10, 2005.

[10]EMC2, "A 15-Minute Guide to Transactional Content Management Foreword," vol. 3, no. 1. New York, pp. 1-10, 2011.

[11]J. E. Scott, "User Perceptions of an Enterprise Content Management System," in Proceedings of the 44th Hawaii International Conference on System Sciences - 2011, 2011, pp. 1-9.

[12]T. C. Chieu, T. Nguyen, and L. Zeng, "Secure Search of Private Documents in an Enterprise Content Management System," IEEE Int. Conf. E-bus. Eng., pp. 105-112, Oct. 2007. 
[13]T. C. Chieu, L. Zeng, and A. Mohindra, "An Extensible Enterprise Content Management System with Service Component Architecture," IBM T. J. Watson Res. Cent., pp. 1131-1137, 2013.

[14]S. Patel, S.K.; Rathod, V.R.; Parikh, "Joomla, Drupal and WordPress - a statistical comparison of open source CMS," Trendz Inf. Sci. Comput., pp. 182-187, 2011.

[15] WebNode, "Sobre Nosotros".[Online], [cited 2015 Feb 19], 2015 Available at: http://www.webnode.es/sobre-nosotros/

[16] WebGUI. "About us" [Online], [cited 2015 Feb 24], 2015, Available from: http://www.webgui.org/

[17]Rubedo. "Presentation". [Online], [cited 2015 Feb 19], 2015, Available from: http://www.rubedoproject.org/home/rubedo/presentation. Accedido [18 02 2015]

[18]ModX, "About us".[Online], [cited 2015 Feb 19], 2015, Available from: http://rtfm.modx.com/
[19] X3CMS, [Online], [cited 2015 Feb 19], 2015, Available at: http://www.x3cms.net/

[20] CMS, [Online], [cited 2015 Feb 19], 2015, Available from: http://cms-software-review.toptenreviews.com/x3-cms-review.html

[21] EE, [Online], [cited 2015 Feb 19], 2015 Available from: http://websmart.tv/learn/what-is-expressionengine/

[22] Movable Type, [Online], [cited 2015 Jan 15], 2015, Available from: https://movabletype.org/about/

[23] Radiant, [Online], [cited 2015 Feb 24], 2015, Available from: http://radiantcms.org/

[24] Youthedesigner. [Online], [cited 2015 Jan 10], 2015, Available from: http://www.youthedesigner.com/graphic-design-tips/review-radiantcms-a-simple-user-friendly-cms/

[25] SilverStripe, [Online], [cited 2015 Feb 19], 2015, Available from: http://www.iwebslog.com/apps/opensource/silverstripe.html 